\title{
Kommentar der Schriftleitung
}

\section{Editor's Comment}

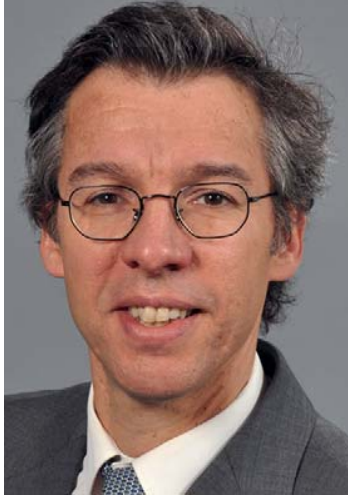

O. Guntinas-Lichius
Bibliografie

DOI http://dx.doi.org/

10.1055/s-0033-1364007

Laryngo-Rhino-Otol 2014; 93:

79 () Georg Thieme

Verlag KG Stuttgart · New York ISSN 0935-8943

Korrespondenzadresse

Prof. Dr. O. Guntinas-Lichius

Klinik und Poliklinik für HNO-

Heilkunde

Universitätsklinikum Jena

Lessingstraße 2

07740 Jena

orlando.guntinas@med.uni-

jena.de
Liebe Leserinnen und Leser,

das Februar-Heft beginnt in der Rubrik „, Referiert und Diskutiert" mit der Diskussion des Einsatzes des ultraschallaktivierten Skalpells als Hilfe bei einer Neck dissection. Die Diskutanten weisen darauf hin, dass möglicherweise der Einsatz zu einem geringeren Blutverlust führt, dies aber genauso wie eine mögliche Zeitersparnis in kontrollierten Studien belegt werden muss.

Die minimal-invasive Therapie ist heute Standard zur Behandlung der Sialolithiasis der Gl. submandibularis. Die Diskutanten sehen dies in Einklang mit den Erfahrungen in Deutschland durch die besprochene aktuelle polnische Arbeit bestätigt [1]. Dazu passt die anschließende Übersicht zur neuen AWMF-Leitlinie zur Behandlung der obstruktiven Sialadenitis. Der Stellenwert der Sialendoskopie wird hier im Einklang mit anderen Diagnostik- und Therapieverfahren von einer interdisziplinären Arbeitsgruppe vorgestellt.

Kein Heft mehr ohne den da Vinci-Roboter $[2,3]$ ?! Dass auch die Kombination von TORS mit einer flexiblen $\mathrm{CO}_{2}$-Laser-Faser funktioniert zeigen uns Kollegen aus Essen.

Kollegen aus Göttingen und Würzburg stellen mit dem „Würzburger Kindersprachtest“ ein Verfahren zur Testung des Sprachverstehens im Störlärm vor. Vielleicht ein wichtiges zukünftiges Diagnostikum bei auditiven Verarbeitungs- und Wahrnehmungsstörungen [4]? Erste Ergebnisse an gesunden Kindern zeigen die Machbarkeit, aber auch eine hohe Streubreite bei Kindern zwischen 5 und 9 Jahren.

Die ambulante Tonsillektomie ist ein heißes Eisen; die aktuelle Diskussion in Österreich bestärkt die Gegner der Ambulantisierung [5-7]. Machen Sie sich selbst ein Bild, ob die Kostenbewertung der ambulanten Tonsillektomie mehr den Gegner als den Befürwortern in die Hand spielt. Der erste Fallbericht behandelt eine laryngeale Sarkoidose. Der Fall ruft in Erinnerung, dass das Chamäleon Sarkoidose eine wichtige wenn auch seltene Differenzialdiagnose des HNO-Fachs darstellt [8]. Im zweiten Fall wird die Behandlung eines fortgeschrittenen Ästhesioneuroblastoms berichtet. Hätte man primär ein PET-CT machen sollen? Hätte man eine primäre neoadjuvante
Therapie vornehmen sollen anstatt primär radikal zu operieren? Was ist Ihre Meinung?

Die ärztliche Aufklärung ist ein wichtiges Thema im HNO-chirurgischen Alltag. Schwerwiegende Risiken gehören aufgeklärt und ein Schaden ist insbesondere dann schwerwiegend wenn dauerhaft und daher muss auf den Dauerschaden bei der Aufklärung unbedingt hingewiesen werden, wie ein aktueller Rechtstreit, präsentiert von Herrn Wienke, eindrücklich belegt.

Die Abklärung einer Dysphagie und vielfach auch die Therapie gehört ins Fachgebiet der HNO-Heilkunde! Daher gehört es auch zum HNO-Facharztwissen, auf den Punkt gebracht durch den Beitrag aus Leipzig am Schluss des Hefts.

Wie immer Ihr Professor Dr. med. O. Guntinas-Lichius Schriftleitung LRO

\section{Literatur}

1 Kroll T, Finkensieper $M$, Hauk $H$, Guntinas-Lichius $O$, Wittekindt $C$. Sialendoskopie - Lernkurve und Verbreitung in deutschen HNO-Kliniken. LaryngoRhino-Otol 2012; 91: 561-565

2 Mattheis S, Mandapathil M, Rothmeier N, Lang S, Dominas N, Hoffmann TK. Transorale Roboter-assistierte Chirurgie von Kopf-Hals-Tumoren: Eine Fallserie mit 17 Patienten. Laryngo-Rhino-Otol 2012; 91: 768-773

3 Lörincz BB, Knecht R. Roboterassistierte Salvage Laryngektomie und Neck Dissection: das Konzept „Robotic Combo Surgery“. Laryngo-Rhino-Otol 2013; 92: $585-588$

4 Nickisch A, Gohde K, Kiese-Himmel C. AVWS bei Regelschülern im 2. Schuljahr: Welche Tests trennen auffällige von unauffälligen Kindern? Laryngo-Rhino-Otol 2013; 92: 594-599

5 Sarny S, Ossimitz G, Habermann W, Stammberger $H$. „Die Österreichische Tonsillenstudie 2010“ - Teil 1: Statistischer Überblick. Laryngo-Rhino-Otol 2012; 91: 16-21

6 Sarny S, Habermann W, Ossimitz G, Stammberger $H$. „Die Österreichische Tonsillenstudie 2010“ - Teil 2: Postoperative Blutungen. Laryngo-Rhino-Otol 2012; 91: 98-102

7 Sarny S, Ossimitz G, Habermann W, Stammberger $H$. Die Österreichische Tonsillenstudie Teil 3: Operationstechnik und Nachblutung nach Tonsillektomie. Laryngo-Rhino-Otol 2013; 92: 92-96

8 Pickhard A, Smith E, Rottscholl R, Brosch S, Reiter R. Manifestation internistischer Erkrankungen im Larynx: Chronisch entzündliche Erkrankungen. Laryngo-Rhino-Otol 2012; 91: 758-766 